\title{
Errata
}

\section{Intermediate Circulation in the Northwestern North Pacific Derived from Subsurface Floats}

Takanori Iwao, Masahiro Endoh, Nobuyuki Shikama and Toshiya Nakano

Journal of Oceanography, Vol. 59, pp. 893-904, 2003

p. 901, captions for Figs. 11 and 12;

"Fig. 11. Total volume transport through each 1 degree grid point in the 26.6-27.0 $\sigma_{\theta}$ layer (contour interval: $1 \mathrm{~Sv} \cdot \mathrm{grid}^{-1}$ ) calculated by accumulation of the product of flow speed and layer thickness of every $0.1 \sigma_{\theta}$ layer from 26.6 to $27.0 \sigma_{\theta}$. The shading indicates the strong flow grid larger than $1 \mathrm{~Sv} \cdot$ grid $^{-1}$. Arrows are the same as yellow arrows in Fig. 9."

should be read as

"Fig. 11. Thickness of the layer of density range 26.6-27.0 $\sigma_{\theta}$ derived from the historical oceanographic observations. Contour interval is $20 \mathrm{~m} . "$

"Fig. 12. Thickness of the layer of density range 26.6-27.0 $\sigma_{\theta}$ derived from the historical oceanographic observations. Contour interval is $20 \mathrm{~m} . "$

should be read as

"Fig. 12. Total volume transport through each 1 degree grid point in the $26.6-27.0 \sigma_{\theta}$ layer (contour interval: $1 \mathrm{~Sv} \cdot \mathrm{grid}^{-1}$ ) calculated by accumulation of the product of flow speed and layer thickness of every $0.1 \sigma_{\theta}$ layer from 26.6 to $27.0 \sigma_{\theta}$. The shading indicates the strong flow grid larger than $1 \mathrm{~Sv} \cdot \mathrm{grid}^{-1}$. Arrows are the same as yellow arrows in Fig. 9." 